\title{
Angular dependence of the depinning field for head-to-head domain walls at constrictions
}

\author{
D. Bedau, M. Kläui, ${ }^{a)}$ and U. Rüdiger \\ Fachbereich Physik, Universität Konstanz, Universitätsstrasse 10, 78457 Konstanz, Germany \\ C. A. F. Vaz ${ }^{\text {b) }}$ and J. A. C. Bland \\ Cavendish Laboratory, University of Cambridge, Madingley Road, Cambridge CB3 OHE, United Kingdom \\ G. Faini and L. Vila ${ }^{\mathrm{c})}$ \\ Laboratoire de Photonique et de Nanostructures-CNRS, Route de Nozay, 91460 Marcoussis, France \\ W. Wernsdorfer \\ Laboratoire L. Néel-CNRS, BP 138, 38274 Grenoble, France
}

\begin{abstract}
The angular dependence of the depinning field of vortex and transverse domain walls is determined experimentally for $\mathrm{NiFe}$ rings with nanometer size constrictions. From the angular dependence, we are able to deduce the potential landscape caused by the constriction and seen by the wall. The potential minimum for transverse walls is at the notch position so that these walls are pinned symmetrically inside the constriction. Vortex walls are pinned at a position adjacent to the constriction and this position can be determined from the angular variation of the depinning fields. Good agreement with the results of micromagnetic simulations is obtained.
\end{abstract}

Recently interest in magnetic domain walls has been surging, fueled by fundamental interest in the magnetic properties, the spin structure and pinning characteristics. Furthermore, devices with switching by field-induced ${ }^{1}$ as well as current-induced $^{1}$ domain wall motions ${ }^{2,3}$ have been suggested.

To control the domain wall behavior and, in particular, the switching, pinning centers can be used to confine the wall propagation in field-induced switching ${ }^{4-6}$ and also in currentinduced switching. ${ }^{3,7}$ Such pinning centers provide welldefined stable locations for domain walls. Pinning centers can result from imperfections in the material, ${ }^{8,9}$ in order to reliably engineer pinning, artificially structured variations in the geometry of an element have been introduced. While protrusions in wires have been used, ${ }^{10,11}$ constrictions have been shown to yield particularly well-defined pinning centers. ${ }^{5,7,12-15}$ In addition to applications, such as domain wall diodes in logic devices, ${ }^{11}$ constrictions have also allowed the determination of more fundamental properties of domain walls, such as magnetoresistance (MR) effects associated with domain walls. $^{13}$

Domain walls move in an effective potential landscape generated by geometrical variations. ${ }^{12-14}$ Rings have proven to be an apt geometry since domain walls can be positioned easily using uniform fields applied along appropriate directions. ${ }^{16}$ In an earlier work, we observed that constrictions create an attractive potential well for transverse walls which are pinned in the constriction [see Fig. 1(c)]. While

\footnotetext{
${ }^{a)}$ Electronic mail: mathias.klaeui@uni-konstanz.de

${ }^{b}$ Present address: Applied Physics, Yale University, New Haven, CT 06520

${ }^{c)}$ Present address: ISSP, University of Tokyo, Kashiwa, Chiba 277-8581, Japan.
}

vortex walls are repelled from a constriction and pinned adjacent to it [see Fig. 1(c)]. The width and depth of the attractive potential well generated by a constriction have been measured along one direction in Refs. 12 and 13. In particular, for vortex walls the field was always applied in a direction to pull the wall away from the constriction, while the field to pull a domain wall across and through a constriction has not been determined. In order to determine the full potential landscape, the depinning field has to be measured for all angles.

In this paper we probe the angular variation of the pinning potential of domain walls at a constriction. The experimentally obtained results are compared with micromagnetic simulations.

The ring structures for this work were fabricated in a two-step lift-off process. The magnetic rings were obtained after deposition and lift-off of Permalloy $(34 \mathrm{~nm})$, followed by a thin gold capping layer $(4 \mathrm{~nm})$. In the second step, contacts were defined again by EBL followed by the lift-off

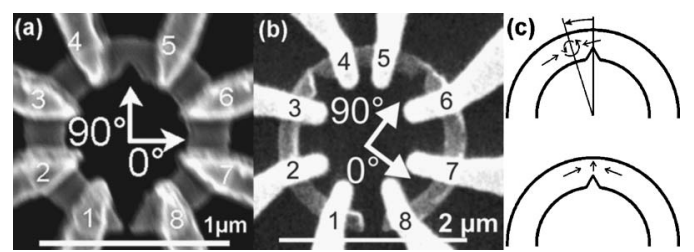

FIG. 1. (a) Scanning electron microscopy (SEM) image of a $34 \mathrm{~nm}$ thick and $200 \mathrm{~nm}$ wide ring with an outer diameter of $1 \mu \mathrm{m}$ and a constriction $(120 \mathrm{~nm})$. Nonmagnetic contacts are positioned on the ring to probe the MR response. In (b) a SEM image of a $34 \mathrm{~nm}$ thick and $200 \mathrm{~nm}$ wide ring with an outer diameter of $2 \mu \mathrm{m}$ and narrower constrictions $(30 \mathrm{~nm})$ is shown. (c) Definition of the angle between the domain wall and the notch, with the notch being located at $90^{\circ}$. Both wall types are shown in their respective equilibrium positions [vortex wall (top) and transverse wall (bottom)]. 


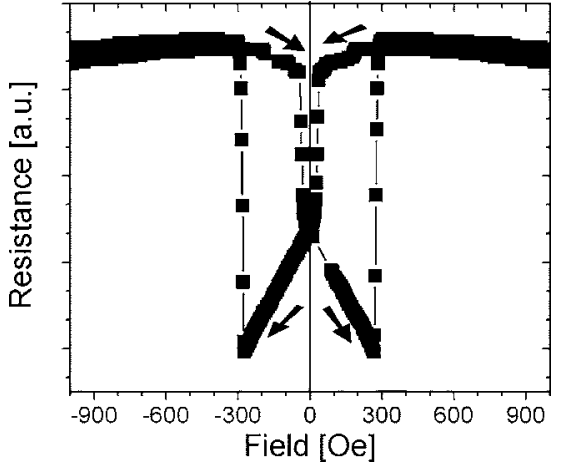

FIG. 2. MR hysteresis loop measured between contacts 4 and 5 of the ring shown in Fig. 1(a) along $20^{\circ}$, showing that the domain wall moves to the position next to the notch upon lowering the field before reaching 0 Oe.

of a Ti $(5 \mathrm{~nm}) / \mathrm{Au}(100 \mathrm{~nm})$ bilayer. The contacts were chosen to be nonmagnetic as otherwise the magnetic states of the ring can be disturbed. Images of such ring structures are presented in Figs. 1(a) and 1(b). Figure 1(c) shows the definition of the angle between the notch and the domain wall used in this text. The MR measurements were done with a current of $10 \mu \mathrm{A}$ using a lock-in technique in a cryostat at temperatures between $35 \mathrm{mK}$ and $4 \mathrm{~K}$ in order to maximize the anisotropic MR (AMR) response and to rule out spin disorder MR. ${ }^{13}$

The position of a domain wall can be sensed by measuring the resistance between two contacts: if a wall is present, the resistance is lower than in the case without a wall. This is due to the magnetization in the domain wall pointing perpendicularly to the current, which lowers the resistance due to the AMR. ${ }^{13}$

To calculate the magnetization configuration, the micromagnetic equilibrium equation was solved for each applied field on a square mesh. ${ }^{17}$ The intrinsic parameters used are $M_{S}=800 \times 10^{3} \mathrm{~A} / \mathrm{m}, \quad A=13 \times 10^{-12} \mathrm{~J} / \mathrm{m}, \quad K_{1}=0.5$ $\times 10^{2} \mathrm{~J} / \mathrm{m}^{3}$, and a cell size of $5 \mathrm{~nm}$.

In wide rings, the natural pinning of domain walls at defects such as edge irregularities is lower than in narrow rings. ${ }^{19}$ Due to the absence of strong pinning, the walls are moved even by a very shallow attractive potential. This means hat very robust switching is possible because the domain wall only needs to be brought somewhere in the vicin- ity of the notch, and at remanence, it will always be located in its equilibrium position adjacent to the notch. It is important to note that the domain wall has to be regarded as an extended object which can therefore experience the potential well of the constriction, even if its center is hundreds of nanometers away. ${ }^{13}$ In Fig. 2 we present a MR measurement taken along $20^{\circ}$, which is $70^{\circ}$ off the notch position. It shows that the resistance drop, which arises from the domain wall moving into the area between contacts 4 and 5, occurs at a field of $+40 \mathrm{Oe}$, which means that it takes a field of $40 \mathrm{Oe}$ along $20^{\circ}$ to balance out the attractive potential well and keep the wall from falling into the pinned position adjacent to the notch. The hysteretic behavior seen in the graph (Fig. 2) shows that once the wall is pinned, it takes a much higher field to depin it again $(\approx 300$ Oe).

When we measure the depinning field after saturating and relaxing the field along different directions in the range from $0^{\circ}$ to $90^{\circ}$, we find that the depinning field is always the same. This indicates that even though the field was applied along different directions, the attractive potential well pulls the domain wall always into the same pinned position, which is also corroborated by the fact that the resistance level observed at 0 Oe is always the same. We now investigate how the depinning field varies with applied field direction. We apply a field along a certain angle and measure the field necessary to depin the domain wall and move it out of the area between the contacts adjacent to the position being investigated.

Figure 3 shows the depinning field for a vortex domain wall located to the left (a) and right (b) of the notch as a function of the angle of the applied field for the ring shown in Fig. 1(a). Between about $60^{\circ}$ and $130^{\circ}$, the field is applied along a direction between contacts 4 and 5, therefore the domain wall stays between these contacts and no switching is observed. As expected the two graphs are mirror symmetric with respect to the $90^{\circ}$ axis $\left[45^{\circ}\right.$ in Fig. 3(b) corresponds to $135^{\circ}$ in Fig. $3(\mathrm{a}), 225^{\circ}$ in Fig. 3(b) corresponds to $315^{\circ}$ in Fig. 3(a), etc.]. A graph showing the simulated switching fields, corresponding to the experimental curve in Fig. 3(a), is shown in Fig. 3(c). The simulations for the angles with thelowest fields yield values very similar to those found experimentally $(\approx 200 \mathrm{Oe})$ and the general trend, including the
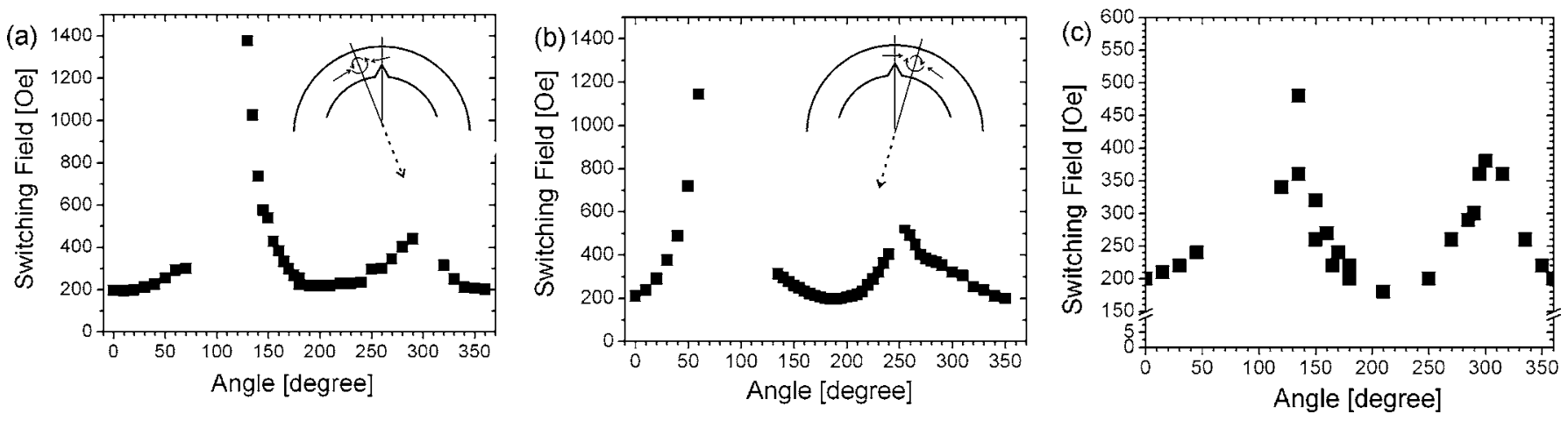

FIG. 3. Graphs showing the switching field (field when the domain wall leaves the area between contacts 4 and 5) of the ring in Fig. 1(a) as a function of the angle of the applied field for (a) the vortex domain wall pinned to the left of the notch (at about $120^{\circ}$ ) and (b) to the right of the notch $\left(\right.$ at about $70^{\circ}$ ). The switching fields are the lowest when the field is approximately perpendicular to the wall position and the highest when the field is opposite (indicated by the dashed arrows in the insets). Graph (c) shows the switching fields calculated using micromagnetic modeling for the case of the domain wall pinned to the left [as in (a)]. 


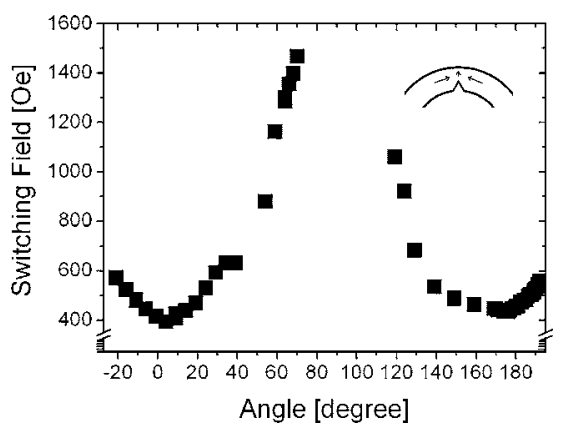

FIG. 4. The angular dependence of the depinning field for the transverse wall is presented. From a maximum around $90^{\circ}$ (notch position, shown in the inset), the depinning field falls off to the minima at around $0^{\circ}$ and $180^{\circ}$. The asymmetry around $90^{\circ}$ is caused by a not perfectly symmetric potential well. Due to the large slope around $90^{\circ}$, a small asymmetry will shift the depinning fields above the maximum field of 1500 Oe used in our experiments. Above $210^{\circ}$ and below $-30^{\circ}$, the reversal does not occur by the depinning of the pinned domain wall, but by the nucleation of a reverse domain.

angles where the maxima and minima of the switching fields are attained, is very well reproduced. In the simulations for angles with higher switching fields, the simulated values are significantly lower than those found experimentally, which indicates that for these values the pinning at defects, which are not included in the simulation, becomes important.

We see that for the domain wall located to the left, a maximum switching field is found for an angle of around $300^{\circ}$ [Fig. 3(a)]. From this we can measure the approximate position of the pinned domain wall, since this maximum of the depinning field is expected to be along the direction approximately opposite to the wall position, as indicated by a dashed arrow in the inset in Figs. 3(a) and 3(b). Thus we find the wall to be positioned at $300^{\circ}-180^{\circ}=120^{\circ}$, in line with our micromagnetic simulations. For the wall located to the right of the notch, the maximum is found at around $245^{\circ}$, which yields a pinned wall position of $245^{\circ}-180^{\circ}=65^{\circ}$.

For idealized pointlike domain wall objects, the acting force on the domain wall would be ${ }^{16,18} H \sin (\theta)$, with $\theta$ the angle between field direction and position of the domain wall (see Fig. 1), and hence the switching field would scale as $H_{c} \propto 1 / \sin (\theta)$. Because the domain wall is an extended object, the switching field always stays below 600 Oe.

For angles between $140^{\circ}$ and $300^{\circ}$, the simulation suggests that the domain wall, which at remanence is located at $120^{\circ}$, leaves the area between contacts 4 and 5 to the left (passing contact 4) and moves down the left half of the ring. For angles between $300^{\circ}$ and $360^{\circ}$ and between $0^{\circ}$ and $70^{\circ}$, the domain wall is pulled through the constriction and leaves the area between the contacts to the right (passing contact 5).

Transverse walls are expected to exhibit a more symmetric angular dependence, since they are pinned inside the constriction. In a ring with a large notch [Fig. 1(b)], the resulting constriction is so narrow that a domain wall is pinned inside the constriction. The walls were determined to be transverse using MR measurements. ${ }^{14}$ This symmetric situation leads to an angular dependence of the depinning fields shown in Fig. 4. Most importantly, we see that the depinning field falls off symmetrically around the notch position $\left(90^{\circ}\right)$, which already shows that the wall is indeed positioned inside the constriction, (as was also demonstrated by direct imaging). ${ }^{12}$

The minima are located approximately at $0^{\circ}$ and $180^{\circ}$, $\left( \pm 90^{\circ}\right.$ off the notch), in line with the assumption that the wall is located symmetrically inside the constriction. As the angle is increased (decreased) further, we find that the depinning field increases again until at above $210^{\circ}$ (below $-30^{\circ}$ ) the reversal does not take place by the depinning of the pinned domain wall, but rather by the nucleation of a new reverse domain at the applied field angle and subsequent domain wall propagation that annihilates the wall located at the constriction. ${ }^{9}$ Such a nucleation occurs at about $650 \mathrm{Oe},{ }^{9}$ and is also confirmed by measurements between different contacts for this sample.

We have investigated the pinning of head-to-head domain walls at a constriction in permalloy rings. We find that domain walls are attracted to the notch from positions at least $70^{\circ}$ away, which might prove useful for applications where reliable switching is of paramount importance. The depinning field exhibits minima at angles perpendicular to the wall position. The maximum is found at an angle opposite to the wall position, which allows us to ascertain the exact wall position, which is in the notch for transverse walls and $25^{\circ}-30^{\circ}$ away from the notch for vortex walls. Asymmetries in the depinning field variation are attributed to whether the wall has to be pulled through the constriction or not. In the case of a transverse wall pinned inside the constriction, a symmetric depinning field is measured. For angles more than $120^{\circ}$ away from the notch position, a crossover of the reversal from wall depinning to the nucleation of a reverse domain occurs.

This work was supported by the Deutsche Forschungsgemeinschaft (SFB 513), the Landesstiftung BadenWürttemberg, the EU ESPRIT program MASSDOTS, the EPSRC and the EU ("Nanocluster Regio Bodensee I: Forschungszusammenarbeit Nanostrukturen"). We would like to thank Edmond Cambril (Laboratoire de Photonique et de Nanostructures-CNRS) for technical support.

${ }^{1}$ D. A. Allwood et al., Science 296, 5575 (2002).

${ }^{2}$ A. Yamaguchi et al., Phys. Rev. Lett. 92, 77205 (2004).

${ }^{3}$ M. Kläui et al., Phys. Rev. Lett. 94, 106601 (2005).

${ }^{4}$ L. Lopez-Diaz et al., Physica B 306, 211 (2001).

${ }^{5}$ C. C. Faulkner et al., J. Appl. Phys. 95, 6717 (2004).

${ }^{6}$ A. Himeno et al., J. Appl. Phys. 93, 8430 (2003).

${ }^{7}$ J. Grollier et al., J. Appl. Phys. 92, 4825 (2002).

${ }^{8}$ J. Grollier et al., Appl. Phys. Lett. 83, 509 (2003).

${ }^{9}$ M. Kläui et al., Appl. Phys. Lett. 84, 951 (2004).

${ }^{10}$ A. Himeno et al., J. Appl. Phys. 97, 66101 (2005).

${ }^{11}$ D. A. Allwood et al., Appl. Phys. Lett. 85, 2848 (2004).

${ }^{12}$ M. Kläui et al., Appl. Phys. Lett. 87, 102509 (2005).

${ }^{13}$ M. Kläui et al., Phys. Rev. Lett. 90, 97202 (2003).

${ }^{14}$ M. Kläui et al., Physica B 343, 343 (2004).

${ }^{15}$ A. J. Zambano and W. P. Pratt, Jr., Appl. Phys. Lett. 85, 1562 (2004).

${ }^{16}$ J. Rothman et al., Phys. Rev. Lett. 86, 1098 (2001).

${ }^{17}$ The freely available oOMMF package can be found at gams.nist.gov/oommf

${ }^{18}$ S. Chikazumi, Physics of Ferromagnetism (Oxford University Press, New York, 1999).

${ }^{19}$ M. Kläui et al., J. Magn. Magn. Mater. 290, 61 (2005). 